\title{
Covalent Triazine Frameworks Incorporating Charged Polypyrrole Channels for High-Performance Lithium-Sulfur Batteries
}

\author{
Jiheon Kim," Ahmed Elabd," Sung-Yoon Chung,* Ali Coskun,* and Jang Wook Choi*
}

\begin{abstract}
Organosulfur polymers have emerged as promising electrode materials for lithium-sulfur ( $\mathrm{Li}-\mathrm{S}$ ) batteries, mainly because of their ability to incorporate and stabilize high sulfur content. The low ionic and electronic conductivity of these polymers, however, limit their cycling performance at high active mass loadings. Moreover, $\mathrm{Li}-$ polysulfide ( $\mathrm{Li}-\mathrm{PS}$ ) shuttling, a fatal phenomenon in the cyclability of $\mathrm{Li}-\mathrm{S}$ batteries, can be mitigated via the entrapment of Li-PS by utilizing various supramolecular interactions. Herein, we report a new approach that incorporates one-dimensional charged polypyrrole into a two-dimensional covalent triazine framework (cPpy-S-CTF) synthesized in the presence of elemental sulfur. The cPpy-S-CTF enabled sulfur loadings up to $83 \mathrm{wt} \%$, thanks to the perfluoroaryl-elemental
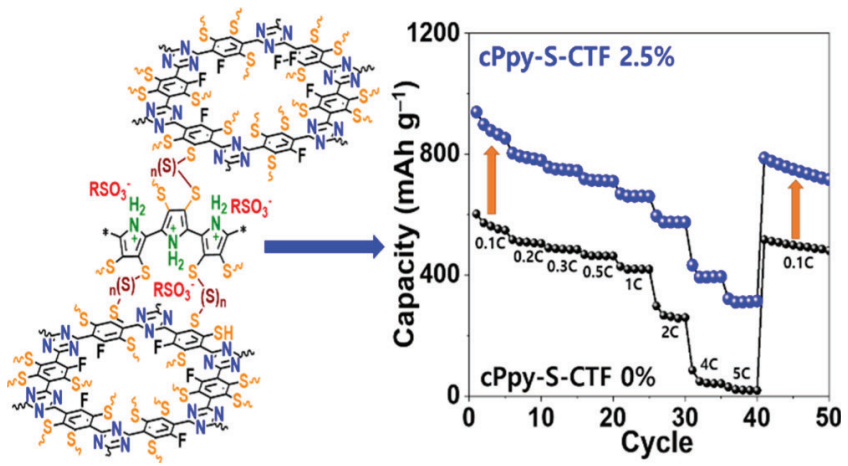
sulfur $\mathrm{SN}_{\mathrm{Ar}}$ chemistry. Notably, the addition of charged polypyrrole, cPpy, triggered a 3D nanochannel formation in the cPpy-S-CTF with high-affinity anchoring sites toward Li-PS, while achieving decent ionic and electronic conductivity. The cPpy-S-CTF showed a remarkable electrochemical performance with a specific capacity of $1203.4 \mathrm{~mA} \mathrm{~h} \mathrm{~g}^{-1}$ at $0.05 \mathrm{C}$, an initial Coulombic efficiency of $94.1 \%$, and a capacity retention of $86.8 \%$ after 500 cycles. These results point to the fact that the incorporation of charged conducting polymers could be a universal strategy to boost the electrochemical performance of organosulfur polymers in Li-S batteries.
\end{abstract}

\section{INTRODUCTION}

The large-scale adaptation of renewable energy in households and public sectors as well as the timely and broad dissemination of electric vehicles necessitate the development of low cost, high-energy density energy storage systems. In this direction, lithium-sulfur ( $\mathrm{Li}-\mathrm{S}$ ) batteries with a high theoretical specific energy density of $2600 \mathrm{~W} \mathrm{~h} \mathrm{~kg}^{-1}$ and the low cost of raw materials have emerged as a promising candidate. In addition, the large-scale utilization of elemental sulfur is highly feasible considering the surplus global supply of sulfur because of its involuntary production from the purification of natural gas and oil by a process called "hydrodesulfurization". ${ }^{1-3}$ Aside from these noticeable characteristics of $\mathrm{Li}-\mathrm{S}$ batteries, there is still a significant gap regarding its broad practical use because of the fact that (1) $\mathrm{Li}-\mathrm{S}$ chemistry is based on solid-liquid-solid transition that involves the insulating solid phases of elemental sulfur and $\mathrm{Li}_{2} \mathrm{~S}$, thus suffering from the interfacial resistance during charging and discharging, (2) the dissolution of lithium polysulfides ( $\mathrm{Li}-\mathrm{PS})$ in the electrolyte results in an inevitable shuttling of lithiated reaction products that impairs the reversibility of discharge-charge in each cycle, ${ }^{4-8}$ and (3) volume expansion up to $80 \%$, which makes it nontrivial to retain the mechanical stability of a sulfur electrode. In order to address these challenges, there have been two main approaches, namely, physical and chemical confinement of sulfur. The first approach involves the utilization of highly porous conducting hosts such as ordered mesoporous carbon (CMK-3), ${ }^{9}$ hierarchical porous carbon, ${ }^{9}$ carbon nanotubes, ${ }^{10}$ porous carbon fibers, ${ }^{11}$ carbon spheres, ${ }^{12}$ graphene, graphene oxide, ${ }^{13-16}$ covalent organic frameworks (COFs) ${ }^{17-19}$ metalorganic frameworks (MOFs), ${ }^{20-23}$ porous organic polymers, ${ }^{24}$ and conducting polymers ${ }^{25-27}$ as hosts for the physical confinement of elemental sulfur. Though these approaches have improved the cycling performance to a large extent, the dissolution of Li-PS and its shuttling due to the lack of specific interactions and the low binding energy between the host and Li-PS led to a capacity decay, especially at high sulfur loadings. ${ }^{28}$ Recently, surface coating of a porous carbon host using a cationic polymer has been shown to alleviate $\mathrm{Li}-\mathrm{PS}$ shuttling. ${ }^{29}$ In addition, in the context of physical confinement, the coating of a conductive layer on the separator ${ }^{30-33}$ or on 
the host material ${ }^{32,34}$ has been demonstrated to increase the electronic and ionic conductivity. ${ }^{35,36}$ The second approach is the chemical confinement based on the strong chemical bonding between sulfur and the polymeric host. This approach is rather promising as it allows the homogeneous distribution and efficient stabilization of sulfur. ${ }^{37-45}$ Having recognized the importance of strong bonding between sulfur and the host material, an inverse vulcanization strategy has been developed to form sulfur-rich copolymers by radical copolymerization of sulfur and organic monomers. ${ }^{46-49}$ While the chemical confinement approach enabled strong binding for sulfur along with a high sulfur content (>80 wt \%), it is still desired to have multiple conducting pathways involving anchoring sites, that is, the heteroatoms and charged sites, to facilitate efficient ionic and electronic transport at substantial sulfur loadings. ${ }^{50}$ Recently, elemental sulfur-mediated synthesis of a covalent triazine framework (CTF) via the trimerization of aromatic nitriles has been reported. ${ }^{51}$ This approach enabled the in situ formation of a conjugated $2 \mathrm{D}$ polymeric host bearing nitrogen atoms as anchoring sites for $\mathrm{Li}^{+}$while achieving a homogeneous distribution of sulfur and its chemical impregnation onto the backbone thorough the radical $\mathrm{C}-\mathrm{H}$ insertion reaction, leading to a sulfur content of $62 \mathrm{wt} \%$. In order to further increase the sulfur content of organosulfur polymers, perfluoroaryl-elemental sulfur nucleophilic aromatic substitution reaction $\left(\mathrm{SN}_{\mathrm{Ar}}\right)$ chemistry was introduced. $^{52,53}$ The synthesis of the organosulfur polymer called SF-CTF was achieved by the trimerization of perfluorinated aryl cyanides with simultaneous impregnation of sulfur, thus enabling achieving sulfur content $>80 \mathrm{wt} \%$. These examples clearly signify the importance of the chemistry of sulfur insertion to achieve a high sulfur content. Because of the limited conductivity of organosulfur polymers, however, further improvements in mass loading, specific capacity, and cycling stability have been hindered.

Supramolecular chemistry, in general, can play a major role in $\mathrm{Li}-\mathrm{S}$ batteries, in particular, for suppressing the $\mathrm{Li}-\mathrm{PS}$ shuttling by engineering specific binding sites targeting $\mathrm{Li}^{+}$or $\mathrm{S}_{n}{ }^{2-}$ or both. In this direction, N,O-doped porous organic polymers, N-doped carbon materials, ${ }^{54-56}$ O-doped mesoporous carbon, ${ }^{57}$ Lewis and acid-base interaction, ${ }^{58-60}$ and COFs based on boronate ester formation have been explored. ${ }^{61}$ However, the ability of these systems to impede the Li-PS shuttling was limited mainly because of the moderate binding strength between $\mathrm{Li}-\mathrm{PS}$ and anchoring sites. In addition, limited sulfur loading and difficulty in fine control of the doping levels further limit the application of these systems. In an effort to mitigate the shuttling effect of $\mathrm{Li}-\mathrm{PS}$, one can benefit from the hard and soft acids and bases (HSAB) theory, which allows predicting which acids and bases prefer to interact. ${ }^{62}$ Specifically, the most favorable acid-base interactions are from hard-hard and soft-soft pairs. The LiPS contains a hard acid, $\mathrm{Li}^{+}$, and a soft base, $\mathrm{S}_{n}{ }^{2-}$, thus targeting $\mathrm{S}_{n}{ }^{2-}$ with a soft acid such as organic cations would be a good direction. All in all, an ideal host material is desired to have (1) high-affinity binding sites toward Li-PS, (2) high ionic/electronic conductivity, (3) sufficient void space to compensate volume expansion, and (4) a high sulfur content (i.e., $>80 \mathrm{wt} \%$ ). With these specifications in mind, herein, we demonstrate the integration of $1 \mathrm{D}$ charged polypyrrole (cPpy) to a $2 \mathrm{D}$ CTF (referred to as cPpy-S-CTF), formed upon the trimerization of tetrafluoroterephthalonitrile in the presence of elemental sulfur and simultaneous sulfur insertion via perfluoroaryl-elemental sulfur $\mathrm{SN}_{\mathrm{Ar}}$ chemistry as well as radical $\mathrm{C}-\mathrm{H}$ insertion onto the cPpy backbone. The choice of $\mathrm{cPpy}$ was based on its unique properties that allow having (1) decent electronic and ionic conductivity, (2) the ability to bind with Li-PS via the HSAB theory, and (3) the capability for releasing the stress of a sulfur composite electrode at high sulfur loadings. The incorporation of $\mathrm{cPpy}^{63-65}$ within the 2D CTF led to the formation of 3D interconnected nanochannels with abundant cationic sites, that is, quaternary nitrogen atoms, as soft acids for efficient $S_{n}{ }^{2-}$ binding as well as for high ionic and electronic conductivity. Notably, cPpy-S-CTF showed a high sulfur content of 83 wt \%. The incorporation of only 2.5 wt \% cPpy improved the electrochemical performance significantly, such as the specific capacity of $1203 \mathrm{~mA} \mathrm{~h} \mathrm{~g}^{-1}$ at $0.05 \mathrm{C}$, initial Coulombic efficiency (ICE) of $94.1 \%$, and capacity retention of $86.8 \%$ after 500 cycles. Also, such robust capacity retention of cPpy-S-CTF was maintained for a high sulfur loading of $4.0 \mathrm{mg}_{\text {sulfur }} \mathrm{cm}^{-2}$.

\section{EXPERIMENTAL SECTION}

Materials. Tetrafluoroterephthalonitrile was purchased from TCI, Japan. 1,3-Dioxolane (DOL), 1,2-dimethoxyethane (DME), elemental sulfur, lithium nitrate $\left(\mathrm{LiNO}_{3}\right)$, lithium bis(trifluoromethane)sulfonamide (LiTFSI), polyvinylidene difluoride (PVDF, $M_{\mathrm{w}}=$ $560,000)$, and polypyrrole were purchased from Sigma-Aldrich, USA. N-Methyl-2-pyrrolidone (NMP) was purchased from Junsei Chemical, Japan. All materials were used without further purification.

Synthesis of cPpy-S-CTFs with Varying Polypyrrole Ratio. The cPpy was incorporated into perfluorinated CTFs to form cPpy-SCTFs by reacting cPpy, tetrafluorophthalonitrile, and elemental sulfur, and the mass ratio of tetrafluorophthalonitrile and cPpy together to elemental sulfur was fixed at 1:3. The cPpy content was varied with respect to the tetrafluorophthalonitrile content in mass ratios of 1:99, 2.5:97.5, 5:95, and 10:90. For example, in the case of cPpy-S-CTF $2.5 \%, 5 \mathrm{mg}$ of cPpy, $0.195 \mathrm{~g}(0.97 \mathrm{mmol})$ of tetrafluorophthalonitrile, and $0.6 \mathrm{~g}$ of elemental sulfur were transferred into a $10 \mathrm{~mL}$ Pyrex ampule. The ampule was purged with $\mathrm{Ar}$ and kept under vacuum while being sealed. The ampule was heated to $160^{\circ} \mathrm{C}$ with a dwelling time of $20 \mathrm{~h}$ at a ramping rate of $1{ }^{\circ} \mathrm{C} \mathrm{min}^{-1}$ in order for the $\mathrm{SN}_{\mathrm{Ar}}$ reaction and trimerization of tetrafluorophthalonitrile to take place. The temperature was raised to $400{ }^{\circ} \mathrm{C}$ with a dwelling time of $20 \mathrm{~h}$ at a ramping rate of $1{ }^{\circ} \mathrm{C} \mathrm{min}{ }^{-1}$ to initiate the inverse vulcanization. The ampule was then cooled down to room temperature producing a black powder to be used for chemical and electrochemical characterization.

Synthesis of cPpy-CTFs. Sulfur-free cPpy-CTFs (0, 2.5, and $10 \%)$ were prepared for the control experiment according to the literature ${ }^{80}$ with modified procedures. In a typical synthesis, cPpy and terephthalonitrile (mass $/$ mass $=0: 100,1: 99,2.5: 97.5,5: 95$, and 10:90) were homogenously mixed with $\mathrm{ZnCl}_{2}$ keeping the mass ratio as 1:5 (cPpy/terephthalonitrile/ $\mathrm{ZnCl}_{2}$ ), and then transferred into a 10 $\mathrm{mL}$ Pyrex ampule. The ampule was sealed under vacuum and heated at $400{ }^{\circ} \mathrm{C}$ for $40 \mathrm{~h}$ with a ramping rate of $1{ }^{\circ} \mathrm{C} \mathrm{min}^{-1}$. Next, the ampule was cooled down to room temperature. The produced black powder was sequentially washed with DI water, $1 \mathrm{M} \mathrm{HCl}$, acetone, and ether to remove reaction residues. The obtained powder was kept in a vacuum oven at $120^{\circ} \mathrm{C}$ overnight for further analyses.

Material Characterization. Fourier transform infrared spectroscopy (FT-IR) analysis was carried out using a TENSOR 27 (Bruker, Germany). X-ray photoelectron spectroscopy (XPS) was performed on an Axis-Supra (KRATOS, United Kingdom) spectrometer. Raman spectra were obtained from a LabRAM HR Evolution (HORIBA, Japan) spectrometer. Thermogravimetric analysis (TGA) was performed on a TGA instrument of Mettler Toledo with a ramping rate of $5{ }^{\circ} \mathrm{C} \mathrm{min}^{-1}$ under a $\mathrm{N}_{2}$ atmosphere in a range of $25-600{ }^{\circ} \mathrm{C}$. Field emission scanning electron microscopy (FESEM) analysis was carried out on a Tescan Mira3 LM FE. Brunauer-Emmett-Teller (BET) analysis was performed on a Micromeritics 3Flex system, and 


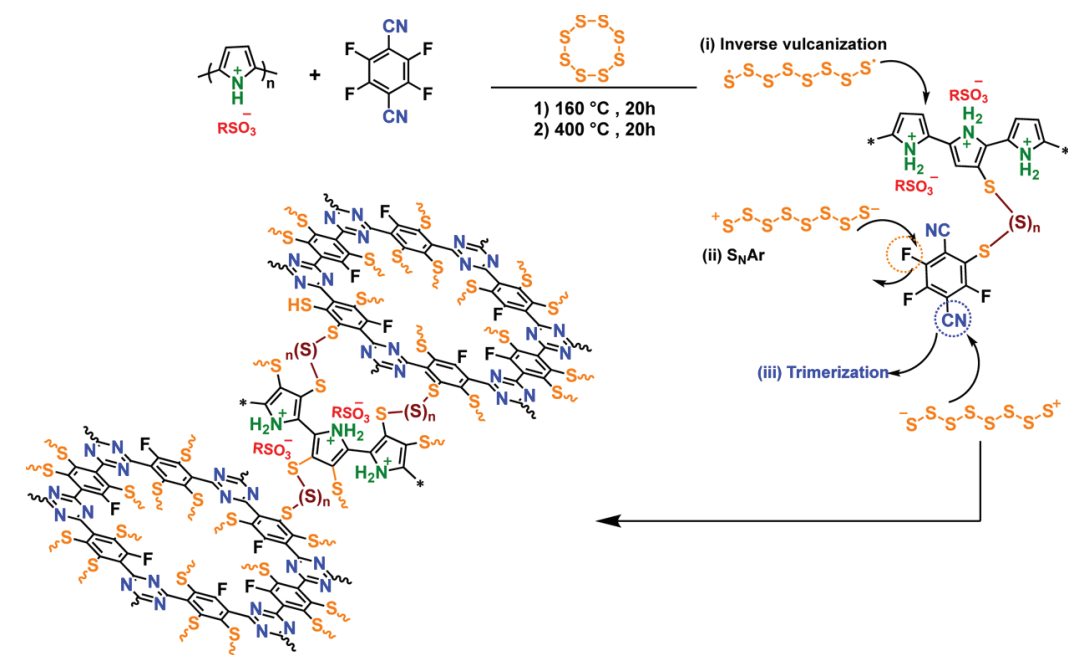

Figure 1. Synthetic scheme for the elemental sulfur-mediated synthesis of cPpy-S-CTFs incorporating different amounts of cPpy via $\mathrm{SN}_{\mathrm{Ar}}$ chemistry and inverse vulcanization.

the surface areas were estimated from $\mathrm{N}_{2}$ adsorption-desorption isotherms using the BET model. The pressure ranges were obtained from the Rouquerol plots. The pore size distributions were calculated using the nonlocal density functional theory (NLDFT) method. Ultraviolet-visible (UV-vis) spectroscopy analysis was performed using a Lambda $35 \mathrm{UV} /$ vis spectrometer (PERKIN ELMER Instruments, USA).

Polysulfide Adsorption Test. To prepare a $50 \mathrm{mM} \mathrm{Li}_{2} \mathrm{~S}_{6}$ solution, stoichiometric amounts of $\mathrm{Li}_{2} \mathrm{~S}$ and elemental sulfur were dissolved in DOL/1,2-dimethoxyethane solution (1:1 in volume ratio) and stirred at $80{ }^{\circ} \mathrm{C}$ for $72 \mathrm{~h}$. The $\mathrm{Li}_{2} \mathrm{~S}_{6}$ solution was then diluted to $3 \mathrm{mM}$ for adsorption tests. All the cPpy-CTFs were dried under vacuum at $60{ }^{\circ} \mathrm{C}$ overnight prior to the adsorption tests.

Electrochemical Characterization of cPpy-S-CTFs. All the battery cells were tested in the form of CR2032-type coin cells. The synthesized composites were ground and mixed with carbon black and PVDF binder in NMP (70:20:10 mass ratio) to form slurries. The prepared slurries were coated onto an aluminum foil $(20 \mathrm{~m}$, Hohsen, Japan) using the doctor blade technique and dried overnight in a convection oven at $60^{\circ} \mathrm{C}$. Each coin cell was assembled in an Ar-filled glove box and a $\mathrm{Li}$ metal disc was used as the counter and reference electrodes. The electrolyte was LiTFSI $(1.0 \mathrm{M})$ and $\mathrm{LiNO}_{3}(0.2 \mathrm{M})$ dissolved in a solvent mixture of $1: 1(\mathrm{v} / \mathrm{v})$ DOL/DME. The polypropylene membrane (Celgard 2400) was used as the separator. The ratio of electrolyte/sulfur was $\sim 15 \mathrm{~mL}_{\text {electrolyte }} \mathrm{g}_{\text {sulfur }}{ }^{-1}$ for all measurements. The galvanostatic tests were programed with a PEBC050.01 battery cycler (PNE Solution, South Korea). The cycling measurements were performed over a voltage range of 1.8-2.7 $\mathrm{V}$ versus $\mathrm{Li} / \mathrm{Li}^{+}$, and electrochemical impedance spectroscopy (EIS) analysis was conducted in the frequency range of $1 \mathrm{MHz}$ to $0.01 \mathrm{~Hz}$ with an amplitude of $10 \mathrm{mV}$ at open circuit potential using VSP (BioLogic, France). Cyclic voltammetry (CV) measurements were conducted between 1.8 and $2.7 \mathrm{~V}$ versus $\mathrm{Li} / \mathrm{Li}^{+}$at a scan rate of $0.05-0.5 \mathrm{mV} \mathrm{s}^{-1}$ by using a WBCS 3000 battery cycler (Wonatech, South Korea). The current densities and specific capacities were all calculated based on sulfur weight only.

\section{RESULTS AND DISCUSSION}

Elemental sulfur-mediated synthesis of covalent triazine frameworks (S-CTF and SF-CTF) enabled the in situ growth of a conjugated polymeric host while simultaneously leading to a homogenous distribution of sulfur and its chemical immobilization onto the backbone. The limited conductivity of CTFs synthesized at $400{ }^{\circ} \mathrm{C}$ and the presence of heteroatoms with a moderate binding affinity toward $\mathrm{Li}^{+}$ through ion-dipole interactions limit the electrochemical performance of these systems. The conductivity issue of CTFs can be lessened markedly by the in situ growth of the conductive polymer during the synthesis of CTFs, which also enables its uniform distribution within the CTF network. In this sense, we identified cPpy as a conductive polymeric additive because of its high ionic and electronic conductivity and the presence of quaternary $\mathrm{N}^{+}$sites offering high binding affinity with $\mathrm{S}_{n}{ }^{2-}$. The synthesis of cPpy-S-CTF was accomplished (Figure 1) via a simple one-step route, that is, reacting tetrafluoroterephthalonitrile and elemental sulfur initially at $160{ }^{\circ} \mathrm{C}$ and afterward at $400{ }^{\circ} \mathrm{C}$ in the presence of different cPpy amounts of $0,1,2.5,5$, and 10 wt \% with respect to tetrafluoroterephthalonitrile weight. The sulfur-tocPpy/monomer ratio was fixed to 3:1 for all the cPpy-S-CTFs. The formation of the CTF backbone through the trimerization of tetrafluoroterephthalonitrile was accompanied by the homogeneous distribution of sulfur and its insertion into both the CTF backbone and cPpy via perfluoroaryl-elemental sulfur $\mathrm{SN}_{\mathrm{Ar}}$ chemistry and radical $\mathrm{C}-\mathrm{H}$ insertion, respectively. As a control experiment, we also synthesized sulfur-free cPpyCTF by synthesizing CTF in the presence of different cPpy amounts of $0,1,2.5,5$, and $10 \mathrm{wt} \%$ (with respect to the weight of terephthalonitrile) under ionothermal reaction conditions at $400{ }^{\circ} \mathrm{C}$ for $40 \mathrm{~h}$ using $\mathrm{ZnCl}_{2}$.

In order to investigate the formation of cPpy-S-CTF, FT-IR analysis was conducted (Figure 2a). The disappearance of the nitrile stretching band at $2218 \mathrm{~cm}^{-1}$ along with the appearance of triazine bands at 1321 and $1509 \mathrm{~cm}^{-1}$ supports the designated synthesis of cPpy-S-CTFs. ${ }^{66}$ The successful integration of cPpy in cPpy-S-CTF was verified by the existence of the quinonoid bipolaron structure of doped cPpy at $973 \mathrm{~cm}^{-1}, 67-69$ which is absent for the S-CTF without cPpy. Moreover, with increasing cPpy content, the stretching vibration of $\mathrm{N}-\mathrm{H}$ was observed at $2986 \mathrm{~cm}^{-1}$, which further proves the addition and structural integrity of cPpy. Importantly, these data collectively suggest that the presence of cPpy in the reaction mixture did not interrupt the formation of 2D CTF. Dispersive Raman analysis was performed (Figure $2 \mathrm{~b}$ ) to probe the formation of $2 \mathrm{D}$ CTF. The appearance of $\mathrm{D}$ and $\mathrm{G}$ bands at 1446 and $1535 \mathrm{~cm}^{-1}$, respectively, indicates the graphitic nature of CTF, which was also reflected in the $I_{\mathrm{D}} / I_{\mathrm{G}}$ ratios close to 1 (Table $S 1$ ). Interestingly, because the $I_{\mathrm{D}} / I_{\mathrm{G}}$ 
(a)

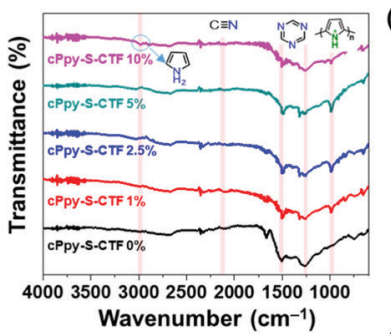

(b)

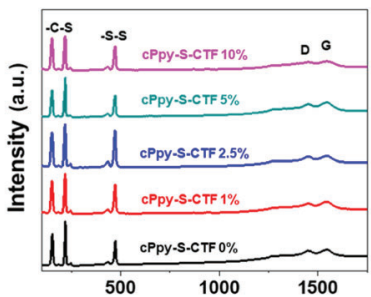

(c)

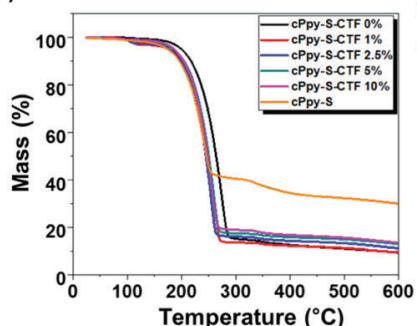

(d)

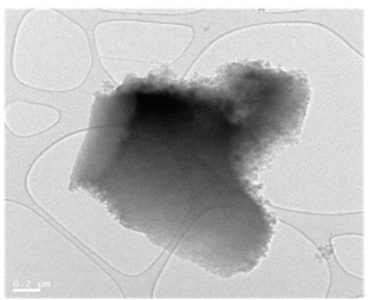

(e)

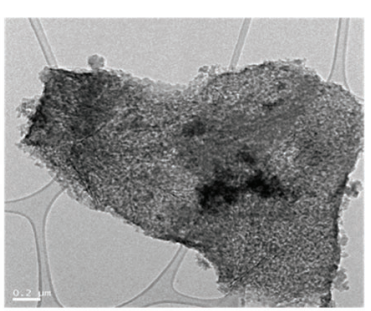

(f)

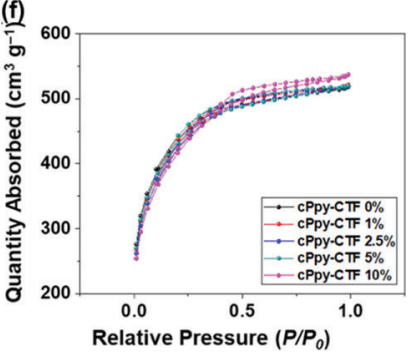

Figure 2. Structural analysis of various cPpy-S-CTFs. (a) FT-IR spectra. (b) Raman spectra. (c) TGA curves obtained under a $\mathrm{N}_{2}$ atmosphere. TEM images of (d) cPpy-S-CTF 0\% and (e) cPpy-SCTF $2.5 \%$. (f) $\mathrm{N}_{2}$ adsorption-desorption isotherms measured at 77 K.

ratio was unaffected by the addition of cPpy, it further indicates the inert nature of cPpy in the formation of CTF. In addition, C-S stretching bands were observed at 234 and 162 $\mathrm{cm}^{-1}$ and the $\mathrm{S}-\mathrm{S}$ band at $464 \mathrm{~cm}^{-1}$, confirming the covalent bonding of sulfur chains to the cPpy and CTF backbones. We also performed (Figure 2c) TGA under a $\mathrm{N}_{2}$ atmosphere to assess the sulfur content of cPpy-S-CTFs with different amounts of cPpy. In all the cPpy-S-CTF series with cPpy contents of $0-10 \mathrm{wt} \%$, the sulfur content was found to be in

the narrow range of $81-83$ wt \%, which points to the insertion of sulfur to the cPpy backbone. TGA analysis of S-cPpy synthesized under the same experimental conditions by reacting cPpy and elemental sulfur showed a relatively low sulfur content of $57 \mathrm{wt} \%$, indicating the importance of $\mathrm{SN}_{\mathrm{Ar}}$ chemistry to accomplish the high sulfur loadings of cPpy-SCTFs. Powder X-ray diffraction (PXRD) analysis (Figure S1) elucidated the presence of physically trapped crystalline sulfur domains within the framework. ${ }^{70}$ Furthermore, the bonding nature of cPpy-S-CTFs was examined (Figures S2 and S3) by XPS over a range of binding energies corresponding to the $\mathrm{C}$ $1 \mathrm{~s}$ and $\mathrm{N} 1 \mathrm{~s}$. The $\mathrm{C} 1 \mathrm{~s}$ spectra of the cPpy-S-CTFs exhibited $\mathrm{C}-\mathrm{C}$ bonding peaks of phenyl and pyrrole moieties at 284.8 $\mathrm{eV} .^{71}$ Their C 1 s spectra also showed $\mathrm{C}-\mathrm{S}$ and triazine peaks at 285.6 and $286.5 \mathrm{eV}$, respectively. ${ }^{52}$ On the other hand, the $\mathrm{C}$ $1 \mathrm{~s}$ spectrum of cPpy showed $\mathrm{C}-\mathrm{C}$ and $\mathrm{C}-\mathrm{N}-\mathrm{H}$ bonds and $\pi-\pi^{*}$ satellite peaks at $284.8,286.2$ and $290.4 \mathrm{eV}$, respectively, which were also observed in cPpy-S-CTFs. ${ }^{72}$ In addition, the N 1s spectra of cPpy-S-CTFs revealed triazine $\mathrm{N}=\mathrm{C}-\mathrm{N}$, pyrrolic $\mathrm{N}-\mathrm{H}$, and $\mathrm{C}-\mathrm{N}^{+}$peaks at $398.4,399.8$, and $401.7 \mathrm{eV}$, respectively. ${ }^{68,73-75}$ These data verify the coexistence of CTF and cPpy in the cPpy-S-CTFs. FE-SEM analysis was performed (Figure S4) in order to elucidate the effect of cPpy on the morphology of cPpy-S-CTFs. Notably, even with the addition of 1 wt \% cPpy, significant changes in the morphology of cPpy$\mathrm{S}$-CTFs were observed. In particular, cPpy-S-CTF $2.5 \%$ showed the most integrated morphology, which is likely to be attributed to the homogeneous distribution of cPpy. However, direct analysis of the distribution of cPpy was infeasible because the signal of cPpy in energy-dispersive X-ray spectroscopy was weak. By contrast, cPpy-S-CTFs with other contents of cPpy displayed particulate or island-like morphology. The formation of the $3 \mathrm{D}$ interconnected structure was verified (Figure $2 \mathrm{~d}, \mathrm{e}$ ) by transmission electron microscopy (TEM) analysis, while cPpy-S-CTF 0\% showed a plain lamellar structure reflective of the $2 \mathrm{D}$ morphology of S-CTF, cPpy-SCTF $2.5 \%$ revealed certain contrast indicating its porous morphology owing to the formation of nanochannels mediated by $\mathrm{cPpy}$. We have also measured (Figure $2 \mathrm{f}$ ) the surface areas of cPpy-CTFs $0,1,2.5,5$, and $10 \%$ using $\mathrm{N}_{2}$ adsorption isotherms measured at $77 \mathrm{~K}$. The surface areas were in the (a)

(b)

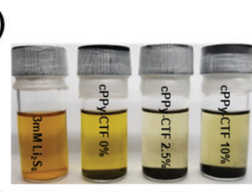

Elapsed time $=3$ hours

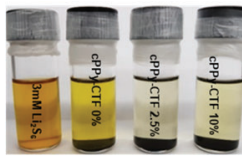

(c)

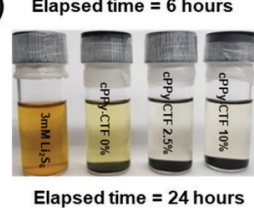

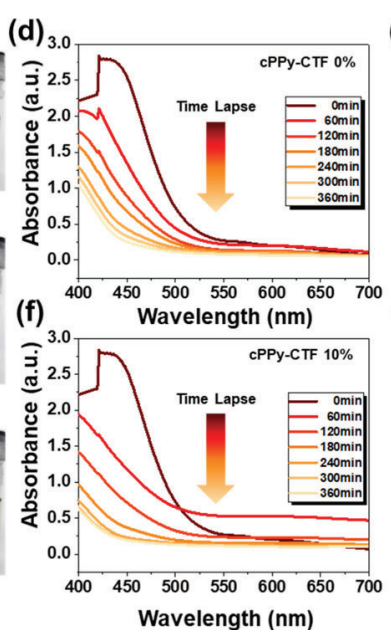

(e)

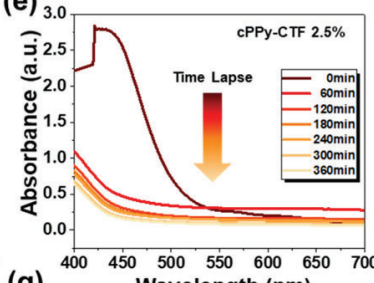

(g)

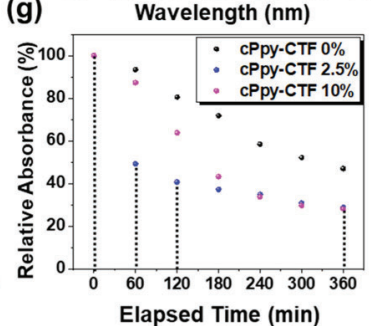

Figure 3. Visual effects of the $3 \mathrm{mM} \mathrm{Li}_{2} \mathrm{~S}_{6}$ in DOL/DME (1:1 v/v) solution when exposed to various cPpy-CTFs over 3, 6, and 24 h (a-c). Timedependent UV-vis absorption spectra of cPpy-CTF 0\% (d), cPpy-CTF $2.5 \%$ (e), and cPpy-CTF 10\% (f) in $3 \mathrm{mM} \mathrm{Li} \mathrm{S}_{6}$ in a DOL/DME (1:1 v/v) solution. (g) Plots of UV-vis absorbance changes with time. 
(a)

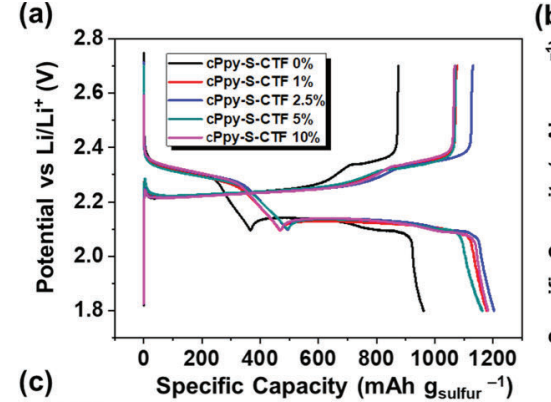

(b)
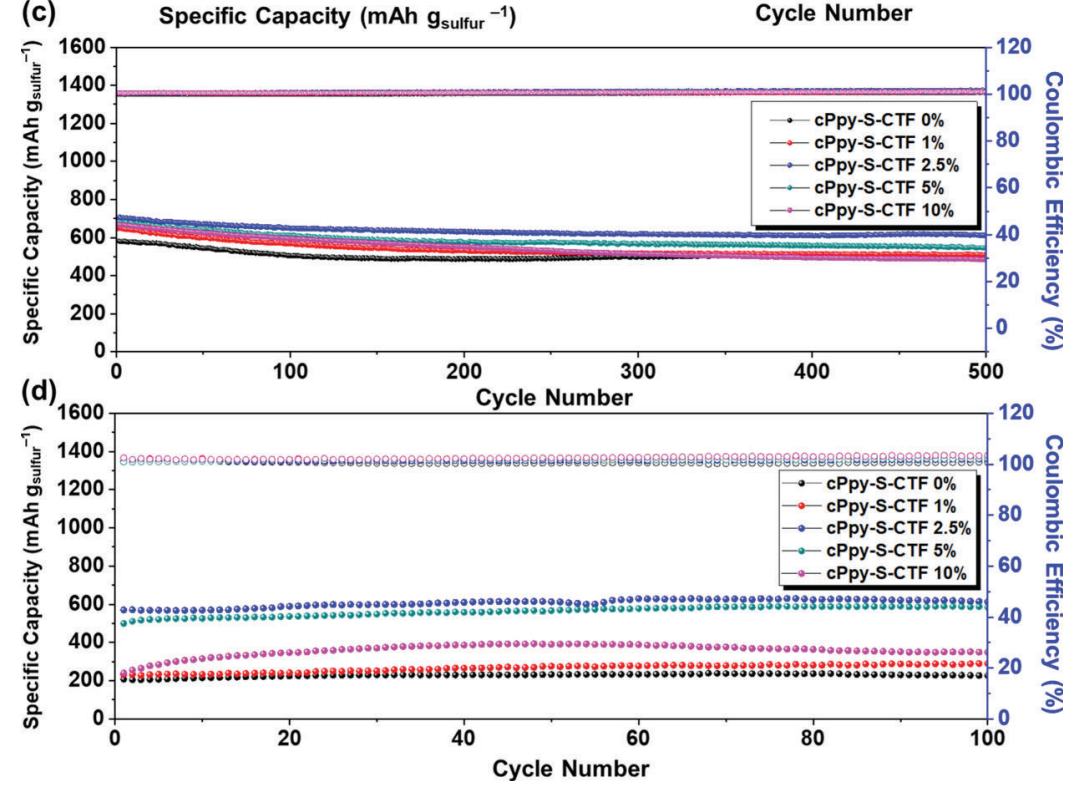

Figure 4. Electrochemical performance of various cPpy-S-CTFs. (a) Comparison of the first discharge-charge profiles of cPpy-S-CTFs at $0.05 \mathrm{C}$ in the potential range $1.8-2.7 \mathrm{~V}\left(1 \mathrm{C}=1675 \mathrm{~mA} \mathrm{~g}^{-1}\right)$. (b) Rate performance of cPpy-S-CTFs $(0-10 \%)$ evaluated at various C-rates $(0.1-5 \mathrm{C})$. (c) Cycling performances and Coulombic efficiencies of cPpy-S-CTFs at $0.5 \mathrm{C}$ and $0.8 \mathrm{mg}_{\text {sulfur }} \mathrm{cm}^{-2}$ for 500 cycles. (d) Cycling performances and Coulombic efficiencies of cPpy-S-CTFs at $0.2 \mathrm{C}$ and $4.0 \mathrm{mg}_{\text {sulfur }} \mathrm{cm}^{-2}$ for 100 cycles.

range of $1483-1561 \mathrm{~m}^{2} \mathrm{~g}^{-1}$ for all cPpy-CTFs (Figures S5-S9 and Table S2), while they all showed type I isotherms pointing to the predominate presence of micropores. Interestingly, we also observed the growth of $\mathrm{H} 4$ hysteresis for cPpy-CTFs with an increase in the cPpy content, which indicates the presence of increased mesopores. This feature was not observed in the absence of cPpy (cPpy-CTF 0\%) because of the efficient stacking of $2 \mathrm{D}$ CTF layers. As expected, increasing the cPpy content slightly decreased the surface area and the micropore content, accompanied by an increase in the mesopore content. The pore size distribution analysis using NLDFT also revealed (Figure S10) a gradual increase in the mesopore content with increasing cPpy amount. In order to probe the impact of cPpy on the Li-PS affinity of the framework, we performed (Figure 3) a polysulfide absorption test by monitoring the timedependent change in the PS concentration using UV-vis spectroscopy. The cPpy-CTF ( $7 \mathrm{mg}$ ) $0,2.5$, and $10 \%$ were added to $3 \mathrm{mM} \mathrm{Li}_{2} \mathrm{~S}_{6}$ in a DOL/1,2-dimethoxyethane (DME) $(1: 1 \mathrm{v} / \mathrm{v})$ solution and monitored for 3,6 , and $24 \mathrm{~h}$ (Figure $3 a-c)$. Notably, the presence of cPpy in cPpy-CTFs substantially improved the Li-PS uptake kinetics (Figure $3 \mathrm{~d}-\mathrm{f}$ ) originating from the strong ionic interactions between a soft acid, the quaternary nitrogen, and a soft base, $\mathrm{S}_{n}{ }^{2-}$. The Li-PS uptake of cPpy-CTFs 1 and 5\% was also compared (Figure S1la-g) with that of cPpy-CTFs 0, 2.5, and $10 \%$. Remarkably, cPpy-CTF $2.5 \%$ showed almost $50 \%$ Li-PS uptake in $1 \mathrm{~h}$, whereas the cPpy-CTFs $0,1,5$, and $10 \%$ showed only $7,2,18$, and $13 \% \mathrm{Li}-\mathrm{PS}$ uptake, respectively, after the same time duration (Figures $3 \mathrm{~g}$ and $\mathrm{S} 11 \mathrm{~g}$ ). This result is rather significant as it shows that there is an optimal spot in the amount of cPpy to realize efficient interactions with the Li-PS. We speculate that the faster uptake kinetics of cPpy-CTF 2.5\% originates from the low aggregation and the homogeneous dispersion of cPpy within the polymer network. Interestingly, the Li-PS uptake of cPpy itself was (Figure S11f,g) lower than that of all the cPpy-CTFs, pointing to the synergistic effect between cPpy and CTF and also to the detrimental role of aggregation of cPpy chains in the Li-PS uptake.

We investigated (Figure 4) the electrochemical performance of cPpy-S-CTFs as active cathode materials in $\mathrm{Li}-\mathrm{S}$ batteries through galvanostatic measurements. For these tests, CR2032type coin cells were prepared by using Li metal as the counter/ reference electrode. The first discharge-charge voltage profiles of cPpy-S-CTF 0, 1, 2.5, 5, and 10\% are shown in Figure 4a when tested at a current density of $0.05 \mathrm{C}(1 \mathrm{C}=1675 \mathrm{~mA}$ $\mathrm{g}^{-1}$ ) with $0.8 \mathrm{mg}_{\text {sulfur }} \mathrm{cm}^{-2}$ loading. We observed discharge capacities of $961.2,1177.6,1203.4,1162.9$, and $1182.6 \mathrm{~mA} \mathrm{~h}$ $\mathrm{g}^{-1}$ and ICE values of 91.1, 91.5, 94.0, 92.2, and 90.3\%, for cPpy-S-CTF 0, 1, 2.5, 5, and 10\%, respectively. Considering the unique properties of cPpy in cPpy-S-CTFs, namely, the high ionic and electronic conductivity as well as the ability to bind with polysulfide anions, we investigated (Figure 4b) their rate performance by invoking different C-rates from 0.1 to $5 \mathrm{C}$ and compared the performances of cPpy-S-CTF 0, 1, 2.5, 5, 
and $10 \%$. The impact of cPpy addition was conspicuous as the rate capability of cPpy-S-CTF 2.5 and $5 \%$ was clearly superior to that of cPpy-S-CTF 0,1 , and $10 \%$. The distinct capacities observed at high C-rates represent a kinetically efficient (de)lithiation process in cPpy-S-CTFs engaging cPpy.

In an attempt to elucidate the effect of cPpy on the cycling performance of cPpy-S-CTFs, these electrodes with $0.8 \mathrm{mg}_{\text {sulfur }}$ $\mathrm{cm}^{-2}$ loading were cycled at $0.5 \mathrm{C}$. Accordingly, the initial discharge capacities of cPpy-S-CTFs $0,1,2.5,5$, and $10 \%$ were found (Figure 4c) to be 582.2, 647.2, 702.9, 683.3, and 667.9

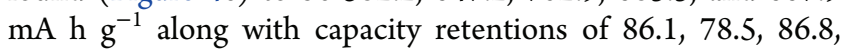
79.8 , and $72.5 \%$ after 500 cycles, respectively. Notably, the cycling performance of cPpy-S-CTF $2.5 \%$ was better than that of all the other samples because of the homogeneous distribution of cPpy and also the accessibility of cationic binding sites for the polysulfide anions, in perfect agreement with the $\mathrm{Li}-\mathrm{PS}$ uptake experiments. In an effort to evaluate the cycling stability at higher $\mathrm{C}$-rates, cyclability tests were carried out (Figure S12) at 1 and $2 \mathrm{C}$ for all cPpy-S-CTFs with $0.8 \mathrm{mg}$ $\mathrm{cm}^{-2}$ of sulfur loading. Notably, cPpy-S-CTF $2.5 \%$ achieved the highest discharge capacities of 699.4 and $631.2 \mathrm{~mA} \mathrm{~h} \mathrm{~g}^{-1}$, respectively, in the initial cycles at 1 and 2 C. cPpy-S-CTF $2.5 \%$ also exhibited superior capacity retentions of 97.6 and $96.7 \%$ after 500 and 300 cycles at 1 and 2 C, respectively. In order to test the electrochemical performance of cPpy-S-CTFs at a higher mass loading, we increased the sulfur loadings to 4.0 $\mathrm{mg}_{\text {suflur }} \mathrm{cm}^{-2}$ and cycled it at $0.2 \mathrm{C}$. The initial discharge capacities of 207.8, 232.2, 570.5, 499.9, and $240.8 \mathrm{~mA} \mathrm{~h} \mathrm{~g}^{-1}$ were lower compared to those at the lower sulfur loading of 0.8 $\mathrm{mg}_{\text {sulfur }} \mathrm{cm}^{-2}$ for cPpy-S-CTF 0, 1, 2.5, 5, and 10\% cPpy, respectively. Nevertheless, cPpy incorporation of 2.5 wt \% resulted in a superior performance compared to all other samples tested, thus re-confirming the beneficial role of cPpy, regardless of the loading amount of the active material. The capacity retention was measured after 100 cycles under the same conditions (Figure 4d). The discharge capacities of the cPpy-S-CTF 0, 1, 2.5, 5, and 10\% after 100 cycles were 227.6, 290.0, 612.9, 583.8, and $348.6 \mathrm{~mA} \mathrm{~h} \mathrm{~g}^{-1}$, respectively. The poor electrochemical performance of cPpy alone (Figure S13) verifies the synergistic effect between cPpy and the CTF backbone.

For attaining an in-depth understanding of the effect of cPpy on the $\mathrm{Li}^{+}$ion diffusion in the framework, we performed $\mathrm{CV}$ measurements (Figure S14) on cPpy-S-CTF 0, 2.5, and 5\% at a scan speed from 0.05 to $0.5 \mathrm{mV} \mathrm{s}^{-1}$. Both cathodic and anodic peak currents were proportional to the square root of the scan speeds, which obeys the following Randles-Sevcik equation $(\text { eq } 1)^{76}$

$$
I_{\mathrm{p}}=\left(2.69 \times 10^{5}\right) n^{1.5} a D^{0.5} v^{0.5} \Delta C_{\mathrm{o}}
$$

where $I_{\mathrm{p}}$ is the peak current, $n$ is the number of electrons per reaction, $a$ is the active electrode area, $D$ is the diffusion coefficient of $\mathrm{Li}^{+}, v$ is the scan velocity, and $\Delta C_{\mathrm{o}}$ is the Li-ion concentration gradient during the electrochemical reaction.

Because $n, a$, and $\Delta C_{\mathrm{o}}$ are constant values, the peak current $I_{\mathrm{p}}$ and the square root of scan velocity $v^{0.5}$ are proportional to each other, and the slope is associated with the square root of diffusivity of the Li ion (Figure S14a-c). Markedly, cPpy-SCTF $2.5 \%$ exhibited the highest slope values for all the cathodic and anodic reactions, which implies that $\mathrm{Li}^{+}$diffusion at this cPpy content is most facile.
We also performed (Figure 5a-c) EIS measurements on cPpy-S-CTF 0\% and cPpy-S-CTF 2.5\%. The Nyquist plots of

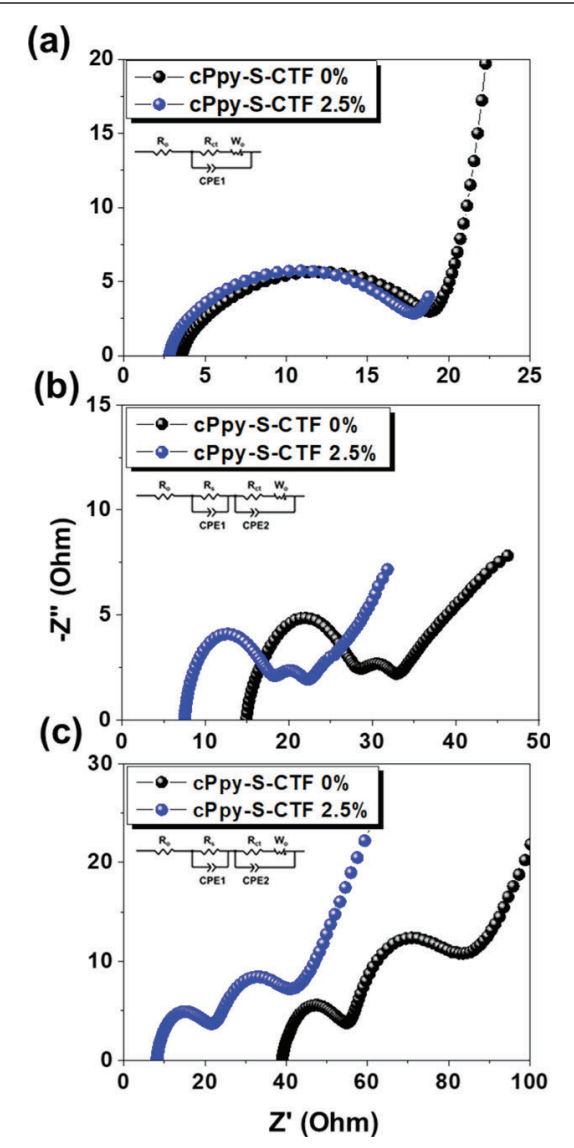

Figure 5. Nyquist plots of cPpy-S-CTF 0 and 2.5\%. (a) Before cycling, (b) after 100th charge, and (c) after 100th discharge.

the two electrodes prior to cycling showed semicircles in the high- and mid-frequency regimes and sloping lines in the lowfrequency regime (Figure 5a). The high-frequency intercept of the real axis represents the bulk resistance $\left(R_{\mathrm{o}}\right)$ of the cell composed of the electrolyte and the electrode resistance. ${ }^{77}$ The semicircle in the middle to the high-frequency regime represents the interface charge transfer resistance $\left(R_{\mathrm{ct}}\right),{ }^{78}$ and the sloping line in the low-frequency regime is related to $\mathrm{Li}$-ion diffusion at the cathode, called Warburg impedance $\left(W_{\mathrm{o}}\right)$. Prior to cycling, $R_{\mathrm{ct}}$ of cPpy-S-CTF $2.5 \%$ was slightly lower than that of cPpy-S-CTF 0\%. After 100 cycles (Figure 5c), cPpy-S-CTF 0\% showed drastically increased bulk resistance compared to that of cPpy-S-CTF 2.5\% (40.3 vs $8.7 \Omega$ ). This phenomenon is attributed to the fact that polysulfide adsorption by cPpy prevents ${ }^{27,79}$ from increasing the viscosity of the electrolyte by $\mathrm{Li}-\mathrm{PS}$ dissolution more effectively compared to the case of cPpy-S-CTF 0\%. Moreover, after cycling, the semicircles of both cells were split into two small semicircles, both of which were clearly smaller for cPpy-S-CTF $2.5 \%$. The semicircle in the high-frequency regime relates to the surface resistance $\left(R_{s}\right)$ corresponding to the deposition on the surface of the electrode and the agglomeration of the $\mathrm{Li}_{2} \mathrm{~S}_{2} / \mathrm{Li}_{2} \mathrm{~S}$ layer. ${ }^{80}$ The semicircle in the intermediate frequency regime relates to the charge transfer process at the conductive interface. ${ }^{81}$ Thus, the smaller semicircles of cPpy-SCTF $2.5 \%$ reflect its lower $R_{\mathrm{s}}$ and $R_{\mathrm{ct}}$ during cycling than that of cPpy-S-CTF 0\%. In order to study the effect of cPpy 
concentration more comprehensively, the EIS data for cPpy-SCTFs with varying cPpy contents were compared (Figure S15). Notably, cPpy-S-CTF $2.5 \%$ exhibited (Table S3) the lowest bulk $\left(R_{\mathrm{o}}\right)$ and interfacial $\left(R_{\mathrm{s}}+R_{\mathrm{ct}}\right)$ resistance among all the cPpy content tested. These results demonstrate that $2.5 \%$ cPpy is the optimal condition for achieving the facile electronic and ionic transport.

\section{CONCLUSIONS}

In summary, we have introduced a $3 \mathrm{D}$ hybrid polymer composite in which a $1 \mathrm{D}$ charged conducting polymer is blended with a sulfur-embedded $2 \mathrm{D}$ conjugated porous polymer network. The in situ formation of the CTF backbone in the presence of elemental sulfur facilitated the homogeneous distribution of sulfur domains alongside cPpy and also enabled the high sulfur content above $80 \%$ through the perfluoroarylelemental sulfur $\mathrm{SN}_{\mathrm{Ar}}$ chemistry. Notably, cPpy incorporation presented not only high affinity cationic anchoring sites for $\mathrm{Li}-$ PS but also well-defined conducting pathways to expedite the Li-ion and electron transport. The homogenous distribution of $2.5 \mathrm{wt} \% \mathrm{cPpy}$ in the new framework was found to be optimal in realizing the efficient binding between soft acid $\mathrm{N}^{+}$and soft base $\mathrm{S}_{n}{ }^{2-}$. In a broader context, this study reveals the potential of supramolecular chemistry in $\mathrm{Li}-\mathrm{S}$ batteries, wherein the optimal binding affinity for polysulfide anions as well as the accessibility of the binding sites are critical in sustaining electrode frameworks and achieving long-term cyclability. In addition, the present strategy represents a major leap for organosulfur polymers suffering from low electronic/ionic conductivity at substantial sulfur mass loadings. Beyond sulfur cathodes, the findings in this study are generally applicable to post-LIB electrodes that undergo large volume changes and unwanted dissolution of reaction products.

\section{ASSOCIATED CONTENT}

\section{ISupporting Information}

The Supporting Information is available

Figures showing structural and porosity analyses results of XPS, PXRD, FESEM, BET linear plots, Rouquerol plots, and NLDFT; additional Li-PS adsorption tests monitored via UV-vis; additional electrochemical analyses; tables presenting the $I_{\mathrm{D}} / I_{\mathrm{G}}$ ratios of CTF, and surface areas and bulk $\left(R_{\mathrm{o}}\right)$ and interfacial $\left(R_{\mathrm{s}}+R_{\mathrm{ct}}\right)$ resistances of cPpy-CTF of various mass ratios (PDF)

\section{AUTHOR INFORMATION}

\section{Corresponding Authors}

Sung-Yoon Chung - Department of Materials Science and Engineering, Korea Advanced Institute of Science and Technology, Daejeon 34141, Republic of Korea; (ㄱ) orcid.org/ 0000-0002-2260-6201; Email: sychung@kaist.ac.kr

Ali Coskun - Department of Chemistry, University of Fribourg, Fribourg 1700, Switzerland; 이이이.org/0000-0002-47601546; Email: ali.coskun@unifr.ch

Jang Wook Choi - School of Chemical and Biological Engineering and Institute of Chemical Processes, Seoul National University, Seoul 08826, Republic of Korea; (1) orcid.org/ 0000-0001-8783-0901; Email: jangwookchoi@snu.ac.kr

\section{Authors}

Jiheon Kim - Department of Materials Science and Engineering, Korea Advanced Institute of Science and Technology, Daejeon 34141, Republic of Korea

Ahmed Elabd - Department of Chemistry, University of Fribourg, Fribourg 1700, Switzerland

Complete contact information is available at:

https://pubs.acs.org/10.1021/acs.chemmater.0c00246

\section{Author Contributions \\ "J.K. and A.E. contributed equally to this work. \\ Notes}

The authors declare no competing financial interest.

\section{ACKNOWLEDGMENTS}

A.C. acknowledges the support from the Swiss National Science Foundation (SNF) for funding of this research (200021-188572). J.W.C. acknowledges financial support from a National Research Foundation of Korea grant (NRF2018R1A2A1A19023146, NRF-2018M1A2A2063340, and NRF-2017M1A2A2044504) and generous support from the Institute of Engineering Research (IER) at Seoul National University.

\section{REFERENCES}

(1) Peramunage, D.; Licht, S. A solid sulfur cathode for aqueous batteries. Science 1993, 261, 1029-1032.

(2) Manthiram, A.; Fu, Y.; Chung, S.-H.; Zu, C.; Su, Y.-S. Rechargeable Lithium-Sulfur Batteries. Chem. Rev. 2014, 114, 11751-11787.

(3) Cleaver, T.; Kovacik, P.; Marinescu, M.; Zhang, T.; Offer, G. Perspective-Commercializing Lithium Sulfur Batteries: Are We Doing the Right Research? J. Electrochem. Soc. 2018, 165, A6029-A6033.

(4) Choi, J. W.; Aurbach, D. Promise and reality of post-lithium-ion batteries with high energy densities. Nat. Rev. Mater. 2016, 1, 16013.

(5) Dong, C.; Gao, W.; Jin, B.; Jiang, Q. Advances in cathode materials for high-performance lithium-sulfur batteries. iScience 2018, 6, 151-198.

(6) Zhu, W.; Paolella, A.; Kim, C.-S.; Liu, D.; Feng, Z.; Gagnon, C.; Trottier, J.; Vijh, A.; Guerfi, A.; Mauger, A.; Julien, C. M.; Armand, M.; Zaghib, K. Investigation of the reaction mechanism of lithium sulfur batteries in different electrolyte systems by in situ Raman spectroscopy and in situ X-ray diffraction. Sustain. Energy Fuels 2017, 1, 737-747.

(7) Wild, M.; O’neill, L.; Zhang, T.; Purkayastha, R.; Minton, G.; Marinescu, M.; Offer, G. J. Lithium sulfur batteries, a mechanistic review. Energy Environ. Sci. 2015, 8, 3477-3494.

(8) Song, Y.; Cai, W.; Kong, L.; Cai, J.; Zhang, Q.; Sun, J. Rationalizing Electrocatalysis of Li-S Chemistry by Mediator Design: Progress and Prospects. Adv. Energy Mater. 2020, 10, 1901075.

(9) Zhang, C.; Wu, H. B.; Yuan, C.; Guo, Z.; Lou, X. W. D. Confining Sulfur in Double-Shelled Hollow Carbon Spheres for Lithium-Sulfur Batteries. Angew. Chem., Int. Ed. 2012, 51, 9592-9595.

(10) Guo, J.; Xu, Y.; Wang, C. Sulfur-Impregnated Disordered Carbon Nanotubes Cathode for Lithium-Sulfur Batteries. Nano Lett. 2011, 11, 4288-4294.

(11) Ji, L.; Rao, M.; Aloni, S.; Wang, L.; Cairns, E. J.; Zhang, Y. Porous carbon nanofiber-sulfur composite electrodes for lithium/ sulfur cells. Energy Environ. Sci. 2011, 4, 5053-5059.

(12) Nan, C.; Lin, Z.; Liao, H.; Song, M.-K.; Li, Y.; Cairns, E. J. Durable Carbon-Coated Li2S Core-Shell Spheres for High Performance Lithium/Sulfur Cells. J. Am. Chem. Soc. 2014, 136, 4659-4663.

(13) Li, B.; Li, S.; Liu, J.; Wang, B.; Yang, S. Vertically Aligned Sulfur-Graphene Nanowalls on Substrates for Ultrafast Lithium-Sulfur Batteries. Nano Lett. 2015, 15, 3073-3079. 
(14) Qiu, Y.; Li, W.; Zhao, W.; Li, G.; Hou, Y.; Liu, M.; Zhou, L.; Ye, F.; Li, H.; Wei, Z.; Yang, S.; Duan, W.; Ye, Y.; Guo, J.; Zhang, Y. High-rate, ultralong cycle-life lithium/sulfur batteries enabled by nitrogen-doped graphene. Nano Lett. 2014, 14, 4821-4827.

(15) Zhou, G.; Pei, S.; Li, L.; Wang, D.-W.; Wang, S.; Huang, K.; Yin, L.-C.; Li, F.; Cheng, H.-M. A Graphene-Pure-Sulfur Sandwich Structure for Ultrafast, Long-Life Lithium-Sulfur Batteries. Adv. Mater. 2014, 26, 625-631.

(16) Park, J.; Kim, E. T.; Kim, C.; Pyun, J.; Jang, H.-S.; Shin, J.; Choi, J. W.; Char, K.; Sung, Y.-E. The Importance of Confined Sulfur Nanodomains and Adjoining Electron Conductive Pathways in Subreaction Regimes of Li-S Batteries. Adv. Energy Mater. 2017, 7, 1700074 .

(17) Liao, H.; Wang, H.; Ding, H.; Meng, X.; Xu, H.; Wang, B.; Ai, $\mathrm{X}$; Wang, C. A $2 \mathrm{D}$ porous porphyrin-based covalent organic framework for sulfur storage in lithium-sulfur batteries. J. Mater. Chem. A 2016, 4, 7416-7421.

(18) Meng, Y.; Lin, G.; Ding, H.; Liao, H.; Wang, C. Impregnation of sulfur into a 2D pyrene-based covalent organic framework for highrate lithium-sulfur batteries. J. Mater. Chem. A 2018, 6, 17186-17191.

(19) Ghazi, Z. A.; Zhu, L.; Wang, H.; Naeem, A.; Khattak, A. M.; Liang, B.; Khan, N. A.; Wei, Z.; Li, L.; Tang, Z. Efficient Polysulfide Chemisorption in Covalent Organic Frameworks for High-Performance Lithium-Sulfur Batteries. Adv. Energy Mater. 2016, 6, 1601250.

(20) Jiang, H.; Liu, X.-C.; Wu, Y.; Shu, Y.; Gong, X.; Ke, F.-S.; Deng, H. Metal-Organic Frameworks for High Charge-Discharge Rates in Lithium-Sulfur Batteries. Angew. Chem., Int. Ed. 2018, 57, 3916-3921.

(21) Zhong, Y.; Xu, X.; Liu, Y.; Wang, W.; Shao, Z. Recent progress in metal-organic frameworks for lithium-sulfur batteries. Polyhedron 2018, 155, 464-484.

(22) Li, F.; Zhang, X.; Liu, X.; Zhao, M. Novel Conductive MetalOrganic Framework for a High-Performance Lithium-Sulfur Battery Host: 2D Cu-Benzenehexathial (BHT). ACS Appl. Mater. Interfaces 2018, 10, 15012-15020.

(23) Jin, J.; Cai, W.; Cai, J.; Shao, Y.; Song, Y.; Xia, Z.; Zhang, Q.; Sun, J. MOF-derived hierarchical CoP nanoflakes anchored on vertically erected graphene scaffolds as self-supported and flexible hosts for lithium-sulfur batteries. J. Mater. Chem. A 2020, 8, 30273034.

(24) Liao, H.; Ding, H.; Li, B.; Ai, X.; Wang, C. Covalent-organic frameworks: potential host materials for sulfur impregnation in lithium-sulfur batteries. J. Mater. Chem. A 2014, 2, 8854-8858.

(25) Choudhury, S.; Agrawal, M.; Formanek, P.; Jehnichen, D.; Fischer, D.; Krause, B.; Albrecht, V.; Stamm, M.; Ionov, L. Nanoporous Cathodes for High-Energy Li-S Batteries from Gyroid Block Copolymer Templates. ACS Nano 2015, 9, 6147-6157.

(26) Yang, Y.; Yu, G.; Cha, J. J.; Wu, H.; Vosgueritchian, M.; Yao, Y.; Bao, Z.; Cui, Y. Improving the Performance of Lithium-Sulfur Batteries by Conductive Polymer Coating. ACS Nano 2011, 5, 91879193.

(27) Fu, Y.; Manthiram, A. Enhanced Cyclability of Lithium-Sulfur Batteries by a Polymer Acid-Doped Polypyrrole Mixed IonicElectronic Conductor. Chem. Mater. 2012, 24, 3081-3087.

(28) Zheng, G.; Zhang, Q.; Cha, J. J.; Yang, Y.; Li, W.; Seh, Z. W.; Cui, Y. Amphiphilic surface modification of hollow carbon nanofibers for improved cycle life of lithium sulfur batteries. Nano Lett. 2013, 13, $1265-1270$.

(29) Li, L.; Pascal, T. A.; Connell, J. G.; Fan, F. Y.; Meckler, S. M.; Ma, L.; Chiang, Y.-M.; Prendergast, D.; Helms, B. A. Molecular understanding of polyelectrolyte binders that actively regulate ion transport in sulfur cathodes. Nat. Commun. 2017, 8, 2277.

(30) Yao, H.; Yan, K.; Li, W.; Zheng, G.; Kong, D.; Seh, Z. W.; Narasimhan, V. K.; Liang, Z.; Cui, Y. Improved lithium-sulfur batteries with a conductive coating on the separator to prevent the accumulation of inactive S-related species at the cathode-separator interface. Energy Environ. Sci. 2014, 7, 3381-3390.

(31) Jin, J.; Wen, Z.; Wang, Q.; Gu, S.; Huang, X.; Chen, C. Protected sulfur cathode with mixed conductive coating layer for lithium sulfur battery. JOM 2016, 68, 2601-2606.
(32) Wang, Q.; Wen, Z.; Yang, J.; Jin, J.; Huang, X.; Wu, X.; Han, J. Electronic and ionic co-conductive coating on the separator towards high-performance lithium-sulfur batteries. J. Power Sources 2016, 306, 347-353.

(33) Yim, T.; Han, S. H.; Park, N. H.; Park, M.-S.; Lee, J. H.; Shin, J.; Choi, J. W.; Jung, Y.; Jo, Y. N.; Yu, J.-S.; Kim, K. J. Effective Polysulfide Rejection by Dipole-Aligned BaTiO3Coated Separator in Lithium-Sulfur Batteries. Adv. Funct. Mater. 2016, 26, 7817-7823.

(34) Jeong, T.-G.; Lee, Y.-S.; Cho, B. W.; Kim, Y.-T.; Jung, H.-G.; Chung, K. Y. Improved performance of dual-conducting polymercoated sulfur composite with high sulfur utilization for lithium-sulfur batteries. J. Alloys Compd. 2018, 742, 868-876.

(35) Milroy, C.; Manthiram, A. An Elastic, Conductive, Electroactive Nanocomposite Binder for Flexible Sulfur Cathodes in Lithium-Sulfur Batteries. Adv. Mater. 2016, 28, 9744-9751.

(36) Yuan, H.; Huang, J.-Q.; Peng, H.-J.; Titirici, M.-M.; Xiang, R.; Chen, R.; Liu, Q.; Zhang, Q. A Review of Functional Binders in Lithium-Sulfur Batteries. Adv. Energy Mater. 2018, 8, 1802107.

(37) Crockett, M. P.; Evans, A. M.; Worthington, M. J. H.; Albuquerque, I. S.; Slattery, A. D.; Gibson, C. T.; Campbell, J. A.; Lewis, D. A.; Bernardes, G. J. L.; Chalker, J. M. Sulfur-Limonene Polysulfide: A Material Synthesized Entirely from Industrial ByProducts and Its Use in Removing Toxic Metals from Water and Soil. Angew. Chem., Int. Ed. 2016, 55, 1714-1718.

(38) Boyd, D. A. Sulfur and its role in modern materials science. Angew. Chem., Int. Ed. 2016, 55, 15486-15502.

(39) Worthington, M. J. H.; Kucera, R. L.; Albuquerque, I. S.; Gibson, C. T.; Sibley, A.; Slattery, A. D.; Campbell, J. A.; Alboaiji, S. F. K.; Muller, K. A.; Young, J.; Adamson, N.; Gascooke, J. R.; Jampaiah, D.; Sabri, Y. M.; Bhargava, S. K.; Ippolito, S. J.; Lewis, D. A.; Quinton, J. S.; Ellis, A. V.; Johs, A.; Bernardes, G. J. L.; Chalker, J. $M$. Laying waste to mercury: inexpensive sorbents made from sulfur and recycled cooking oils. Chem. Eur. J. 2017, 23, 16219-16230.

(40) Je, S. H.; Buyukcakir, O.; Kim, D.; Coskun, A. Direct utilization of elemental sulfur in the synthesis of microporous polymers for natural gas sweetening. Chem 2016, 1, 482-493.

(41) Parker, D. J.; Jones, H. A.; Petcher, S.; Cervini, L.; Griffin, J. M.; Akhtar, R.; Hasell, T. Low cost and renewable sulfur-polymers by inverse vulcanisation, and their potential for mercury capture. J. Mater. Chem. A 2017, 5, 11682-11692.

(42) Kim, J.-S.; Hwang, T. H.; Kim, B. G.; Min, J.; Choi, J. W. A Lithium-Sulfur Battery with a High Areal Energy Density. Adv. Funct. Mater. 2014, 24, 5359-5367.

(43) Hwang, T. H.; Jung, D. S.; Kim, J.-S.; Kim, B. G.; Choi, J. W. One-Dimensional Carbon-Sulfur Composite Fibers for Na-S Rechargeable Batteries Operating at Room Temperature. Nano Lett. 2013, 13, 4532-4538.

(44) Wang, M.; Song, Y.; Sun, Z.; Shao, Y.; Wei, C.; Xia, Z.; Tian, Z.; Liu, Z.; Sun, J. Conductive and Catalytic VTe2@MgO Heterostructure as Effective Polysulfide Promotor for Lithium-Sulfur Batteries. ACS Nano 2019, 13, 13235-13243.

(45) Song, Y.; Zhao, W.; Kong, L.; Zhang, L.; Zhu, X.; Shao, Y.; Ding, F.; Zhang, Q.; Sun, J.; Liu, Z. Synchronous immobilization and conversion of polysulfides on a $\mathrm{VO} 2-\mathrm{VN}$ binary host targeting high sulfur load Li-S batteries. Energy Environ. Sci. 2018, 11, 2620-2630.

(46) Chung, W. J.; Griebel, J. J.; Kim, E. T.; Yoon, H.; Simmonds, A. G.; Ji, H. J.; Dirlam, P. T.; Glass, R. S.; Wie, J. J.; Nguyen, N. A.; Guralnick, B. W.; Park, J.; Somogyi, Á.; Theato, P.; Mackay, M. E.; Sung, Y.-E.; Char, K.; Pyun, J. The use of elemental sulfur as an alternative feedstock for polymeric materials. Nat. Chem. 2013, 5, 518. (47) Je, S. H.; Hwang, T. H.; Talapaneni, S. N.; Buyukcakir, O.; Kim, H. J.; Yu, J.-S.; Woo, S.-G.; Jang, M. C.; Son, B. K.; Coskun, A.; Choi, J. W. Rational Sulfur Cathode Design for Lithium-Sulfur Batteries: Sulfur-Embedded Benzoxazine Polymers. ACS Energy Lett 2016, 1, 566-572.

(48) Simmonds, A. G.; Griebel, J. J.; Park, J.; Kim, K. R.; Chung, W. J.; Oleshko, V. P.; Kim, J.; Kim, E. T.; Glass, R. S.; Soles, C. L.; Sung, Y.-E.; Char, K.; Pyun, J. Inverse Vulcanization of Elemental Sulfur to 
Prepare Polymeric Electrode Materials for Li-S Batteries. ACS Macro Lett. 2014, 3, 229-232.

(49) Kim, H.; Lee, J.; Ahn, H.; Kim, O.; Park, M. J. Synthesis of three-dimensionally interconnected sulfur-rich polymers for cathode materials of high-rate lithium-sulfur batteries. Nat. Commun. 2015, 6, 7278.

(50) Zeng, Z.; Liu, X. Sulfur Immobilization by "Chemical Anchor" to Suppress the Diffusion of Polysulfides in Lithium-Sulfur Batteries. Adv. Mater. Interfaces 2018, 5, 1701274.

(51) Talapaneni, S. N.; Hwang, T. H.; Je, S. H.; Buyukcakir, O.; Choi, J. W.; Coskun, A. Elemental-Sulfur-Mediated Facile Synthesis of a Covalent Triazine Framework for High-Performance Lithium-Sulfur Batteries. Angew. Chem., Int. Ed. 2016, 55, 3106-3111.

(52) Je, S. H.; Kim, H. J.; Kim, J.; Choi, J. W.; Coskun, A. Perfluoroaryl-Elemental Sulfur SN Ar Chemistry in Covalent Triazine Frameworks with High Sulfur Contents for Lithium-Sulfur Batteries. Adv. Funct. Mater. 2017, 27, 1703947.

(53) Shin, H.; Kim, D.; Kim, H. J.; Kim, J.; Char, K.; Yavuz, C. T.; Choi, J. W. Fluorinated Covalent Organic Polymers for High Performance Sulfur Cathodes in Lithium-Sulfur Batteries. Chem. Mater. 2019, 31, 7910-7921.

(54) Song, J.; Gordin, M. L.; Xu, T.; Chen, S.; Yu, Z.; Sohn, H.; Lu, J.; Ren, Y.; Duan, Y.; Wang, D. Strong Lithium Polysulfide Chemisorption on Electroactive Sites of Nitrogen-Doped Carbon Composites For High-Performance Lithium-Sulfur Battery Cathodes. Angew. Chem., Int. Ed. 2015, 54, 4325-4329.

(55) Schneider, A.; Weidmann, C.; Suchomski, C.; Sommer, H.; Janek, J.; Brezesinski, T. Ionic Liquid-Derived Nitrogen-Enriched Carbon/Sulfur Composite Cathodes with Hierarchical Microstructure-A Step Toward Durable High-Energy and High-Performance Lithium-Sulfur Batteries. Chem. Mater. 2015, 27, 1674-1683.

(56) Tang, C.; Zhang, Q.; Zhao, M.-Q.; Huang, J.-Q.; Cheng, X.-B.; Tian, G.-L.; Peng, H.-J.; Wei, F. Nitrogen-Doped Aligned Carbon Nanotube/Graphene Sandwiches: Facile Catalytic Growth on Bifunctional Natural Catalysts and Their Applications as Scaffolds for HighRate Lithium-Sulfur Batteries. Adv. Mater. 2014, 26, 6100-6105.

(57) Demir-Cakan, R.; Morcrette, M.; Nouar, F.; Davoisne, C.; Devic, T.; Gonbeau, D.; Dominko, R.; Serre, C.; Férey, G.; Tarascon, J.-M. Cathode Composites for Li-S Batteries via the Use of Oxygenated Porous Architectures. J. Am. Chem. Soc. 2011, 133, 16154-16160.

(58) Zheng, J.; Tian, J.; Wu, D.; Gu, M.; Xu, W.; Wang, C.; Gao, F.; Engelhard, M. H.; Zhang, J.-G.; Liu, J.; Xiao, J. Lewis Acid-Base Interactions between Polysulfides and Metal Organic Framework in Lithium Sulfur Batteries. Nano Lett. 2014, 14, 2345-2352.

(59) Zhou, J.; Li, R.; Fan, X.; Chen, Y.; Han, R.; Li, W.; Zheng, J.; Wang, B.; Li, X. Rational design of a metal-organic framework host for sulfur storage in fast, long-cycle Li-S batteries. Energy Environ. Sci. 2014, 7, 2715-2724.

(60) Kwon, T.-w.; Choi, J. W.; Coskun, A. Prospect for Supramolecular Chemistry in High-Energy-Density Rechargeable Batteries. Joule 2019, 3, 662.

(61) Ghazi, Z. A.; Zhu, L.; Wang, H.; Naeem, A.; Khattak, A. M.; Liang, B.; Khan, N. A.; Wei, Z.; Li, L.; Tang, Z. Efficient Polysulfide Chemisorption in Covalent Organic Frameworks for High-Performance Lithium-Sulfur Batteries. Adv. Energy Mater. 2016, 6, 1601250.

(62) Pearson, R. G. Hard and soft acids and bases. J. Am. Chem. Soc. 1963, 85, 3533-3539.

(63) Yin, F.; Liu, X.; Zhang, Y.; Zhao, Y.; Menbayeva, A.; Bakenov, Z.; Wang, X. Well-dispersed sulfur anchored on interconnected polypyrrole nanofiber network as high performance cathode for lithium-sulfur batteries. Solid State Sci. 2017, 66, 44-49.

(64) Liu, X.; Qian, T.; Liu, J.; Tian, J.; Zhang, L.; Yan, C. Greatly Improved Conductivity of Double-Chain Polymer Network Binder for High Sulfur Loading Lithium-Sulfur Batteries with a Low Electrolyte/Sulfur Ratio. Small 2018, 14, 1801536.

(65) Wang, J.; Lu, L.; Shi, D.; Tandiono, R.; Wang, Z.; Konstantinov, K.; Liu, H. A Conductive Polypyrrole-Coated, Sulfur-
Carbon Nanotube Composite for Use in Lithium-Sulfur Batteries. ChemPlusChem 2013, 78, 318-324.

(66) Kuhn, P.; Antonietti, M.; Thomas, A. Porous, Covalent Triazine-Based Frameworks Prepared by Ionothermal Synthesis. Angew. Chem., Int. Ed. 2008, 47, 3450-3453.

(67) Chougule, M. A.; Pawar, S. G.; Godse, P. R.; Mulik, R. N.; Sen, S.; Patil, V. B. Synthesis and characterization of polypyrrole (PPy) thin films. Soft Nanosci. Lett. 2011, 01, 6.

(68) Su, N.; Li, H.; Yuan, S.; Yi, S.; Yin, E. Synthesis and characterization of polypyrrole doped with anionic spherical polyelectrolyte brushes. eXPRESS Polym. Lett. 2012, 6, 697.

(69) Yuan, X.; Zeng, X.; Zhang, H.-J.; Ma, Z.-F.; Wang, C.-Y. Improved Performance of Proton Exchange Membrane Fuel Cells withp-Toluenesulfonic Acid-Doped Co-PPy/C as Cathode Electrocatalyst. J. Am. Chem. Soc. 2010, 132, 1754-1755.

(70) Smith, J. A.; Wu, X.; Berry, N. G.; Hasell, T. High sulfur content polymers: The effect of crosslinker structure on inverse vulcanization. J. Polym. Sci. Pol. Chem. 2018, 56, 1777-1781.

(71) Li, Z.; Zhang, S.; Zhang, C.; Ueno, K.; Yasuda, T.; Tatara, R.; Dokko, K.; Watanabe, M. One-pot pyrolysis of lithium sulfate and graphene nanoplatelet aggregates: in situ formed Li2S/graphene composite for lithium-sulfur batteries. Nanoscale 2015, 7, 1438514392.

(72) Nam, D.-H.; Kim, M.-J.; Lim, S.-J.; Song, I.-S.; Kwon, H.-S. Single-step synthesis of polypyrrole nanowires by cathodic electropolymerization. J. Mater. Chem. A 2013, 1, 8061-8068.

(73) Ruangchuay, L.; Schwank, J.; Sirivat, A. Surface degradation of $\alpha$-naphthalene sulfonate-doped polypyrrole during XPS characterization. Appl. Surf. Sci. 2002, 199, 128-137.

(74) Tabačiarová, J.; Mičušík, M.; Fedorko, P.; Omastová, M. Study of polypyrrole aging by XPS, FTIR and conductivity measurements. Polym. Degrad. Stabl. 2015, 120, 392-401.

(75) Wei, Y.; Li, L.; Yang, X.; Pan, G.; Yan, G.; Yu, X. One-Step UVInduced Synthesis of Polypyrrole/Ag Nanocomposites at the Water/ Ionic Liquid Interface. Nanoscale Res. Lett. 2009, 5, 433.

(76) Gewirth, A. A. Inorganic Electrochemistry: Theory, Practice and Application By Piero Zanello (University of Siena, Italy). Royal Society of Chemistry: Cambridge. 2003. xiv +616 pp. $\$ 199.00$. ISBN 0-85404-661-5. J. Am. Chem. Soc. 2004, 126, 4743-4744.

(77) Zhang, S. S.; Xu, K.; Jow, T. R. Electrochemical impedance study on the low temperature of Li-ion batteries. Electrochim. Acta 2004, 49, 1057-1061.

(78) Kolosnitsyn, V. S.; Kuzmina, E. V.; Karaseva, E. V.; Mochalov, $\mathrm{S}$. E. A study of the electrochemical processes in lithium-sulphur cells by impedance spectroscopy. J. Power Sources 2011, 196, 1478-1482.

(79) Fu, Y.; Manthiram, A. Orthorhombic Bipyramidal Sulfur Coated with Polypyrrole Nanolayers As a Cathode Material for Lithium-Sulfur Batteries. J. Phys. Chem. C 2012, 116, 8910-8915.

(80) Zhang, Z.; Kong, L.-L.; Liu, S.; Li, G.-R.; Gao, X.-P. A HighEfficiency Sulfur/Carbon Composite Based on 3D Graphene Nanosheet@Carbon Nanotube Matrix as Cathode for Lithium-Sulfur Battery. Adv. Energy Mater. 2017, 7, 1602543.

(81) Zhang, X.-Q.; Sun, Q.; Dong, W.; Li, D.; Lu, A.-H.; Mu, J.-Q.; Li, W.-C. Synthesis of superior carbon nanofibers with large aspect ratio and tunable porosity for electrochemical energy storage. J. Mater. Chem. A 2013, 1, 9449-9455. 